\title{
Conventional DCs reduce liver ischemia/ reperfusion injury in mice via IL-10 secretion
}

\author{
Zubin M. Bamboat, Lee M. Ocuin, Vinod P. Balachandran, Hebroon Obaid, \\ George Plitas, and Ronald P. DeMatteo
}

Hepatopancreatobiliary Service, Memorial Sloan-Kettering Cancer Center (MSKCC), New York, New York, USA.

\begin{abstract}
TLRs are recognized as promoters of tissue damage, even in the absence of pathogens. TLR binding to damage-associated molecular patterns (DAMPs) released by injured host cells unleashes an inflammatory cascade that amplifies tissue destruction. However, whether TLRs possess the reciprocal ability to curtail the extent of sterile inflammation is uncertain. Here, we investigated this possibility in mice by studying the role of conventional DCs (cDCs) in liver ischemia/reperfusion (I/R) injury, a model of sterile inflammation. Targeted depletion of mouse $c D C$ s increased liver injury after $I / R$, as assessed by serum alanine aminotransferase and histologic analysis. In vitro, we identified hepatocyte DNA as an endogenous ligand to TLR9 that promoted cDCs to secrete IL-10. In vivo, $\mathrm{CDC}$ production of IL-10 required TLR9 and reduced liver injury. In addition, we found that inflammatory monocytes recruited to the liver via chemokine receptor 2 were downstream targets of cDC IL-10. IL-10 from cDCs reduced production of TNF, IL-6, and ROS by inflammatory monocytes. Our results implicate inflammatory monocytes as mediators of liver $I / R$ injury and reveal that $c D C s$ respond to DAMPS during sterile inflammation, providing the host with protection from progressive tissue damage.
\end{abstract}

\section{Introduction}

Optimal clearance of bacterial, viral, and parasitic pathogens is a delicate balance between engaging an inflammatory response and preventing unnecessary damage to healthy tissues. In the absence of infection, insults such as trauma, hemorrhage, or ischemia/reperfusion (I/R) can trigger the release of endogenous damage-associated molecular patterns (DAMPs), which may precipitate a dysregulated immune response through their interaction with pattern recognition receptors (1). DAMPs can be classified as intracellular proteins, nucleic acids, or components of the extracellular matrix that are released following cellular injury. Of the various pattern recognition receptors on immune cells, those of the TLR family are the most well characterized and extensively studied. Collateral tissue damage during sterile injury stems from the inability of TLRs to distinguish infectious ligands from DAMPs released by autologous tissue. As a result, similar downstream pathways are triggered to those that are during infection, and a proinflammatory response is initiated.

Liver I/R injury is a clinically relevant condition, in which the host response to DAMPs induces exuberant immune activation, resulting in hepatocyte death and ultimately liver failure. I/R injury occurs during organ transplantation, hepatic resection, and various forms of circulatory shock. It contributes to the shortage of organs available for transplantation and is a major determinant of postoperative allograft dysfunction and morbidity (2). The need for effective approaches to manage patients with I/R-induced organ damage is highlighted by the fact that current treatment is merely supportive care.

The innate immune response to DAMPs, such as high-mobility group box 1 (HMGB1), RNA, and heat shock proteins, has traditionally been recognized as proinflammatory (3-5). A slew of cytokines and chemokines, including TNF, IL-6, and monocyte chemoattractant protein-1 (MCP-1), are secreted following hepatic ischemia and contribute to the pathogenesis of liver I/R (6-8). Early

Conflict of interest: The authors have declared that no conflict of interest exists. Citation for this article: J Clin Invest. 2010;120(2):559-569. doi:10.1172/JCI40008. activation of Kupffer cells, endothelia, and the complement cascade during liver I/R promotes neutrophil recruitment and the generation of ROS that mediate additional cell death (9). While the proinflammatory signaling cascades and cellular mediators of $\mathrm{I} / \mathrm{R}$ injury have been studied extensively, there are scant data on mechanisms that limit the immune response. Cytokines, such as IL-4, IL-10, and IL-13, have been shown to play a protective role in rodent models of I/R, but the cells and pathways involved remain unresolved (10-12).

In contrast to other nonlymphoid organs, the liver is enriched in leukocytes and is continually faced with the challenge of differentiating harmless ingested antigens from potentially lethal bacterial toxins that translocate from the gut. Although the liver can mount an appropriate and at times exuberant immune response, the overall liver balance appears to favor a state of immune permissiveness. As critical regulators of both innate and adaptive immunity, hepatic DCs are poised to orchestrate immune responses in a manner that limits undesirable inflammation and promotes tolerance (13). Evidence of the regulatory role of hepatic DCs comes from work in murine models of liver transplantation in which donor-derived DCs are thought to contribute to allograft acceptance $(13,14)$.

We hypothesized that under conditions of sterile inflammation, hepatic DCs respond to DAMPs in a manner that limits host tissue damage. Using a model of segmental liver ischemia in transgenic CD11c-diphtheria toxin receptor (DTR) mice, we identified conventional DCs (cDCs) as critical modulators of the inflammatory response during liver I/R injury. Hepatocyte DNA activated hepatic cDCs via TLR9 to secrete IL-10. Moreover, we identified inflammatory monocytes as mediators of liver injury that are recruited to the ischemic liver via chemokine receptor 2-mediated (CCR2mediated) egress from the bone marrow. Production of IL-10 by cDCs during I/R suppressed inflammatory monocyte function and reduced liver injury.

\section{Results}

Liver $c D C$ s are depleted during $I / R$. To determine the effects of hepatic $\mathrm{I} / \mathrm{R}$ on liver $\mathrm{cDCs}\left(\mathrm{CD} 11 \mathrm{c}^{\text {hi }} \mathrm{MHCII}{ }^{\mathrm{hi}}\right)$, we examined $\mathrm{CD} 45^{+}$nonparen- 
A

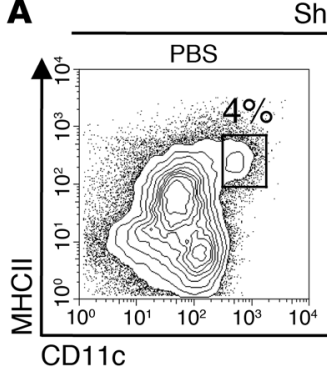

Sham
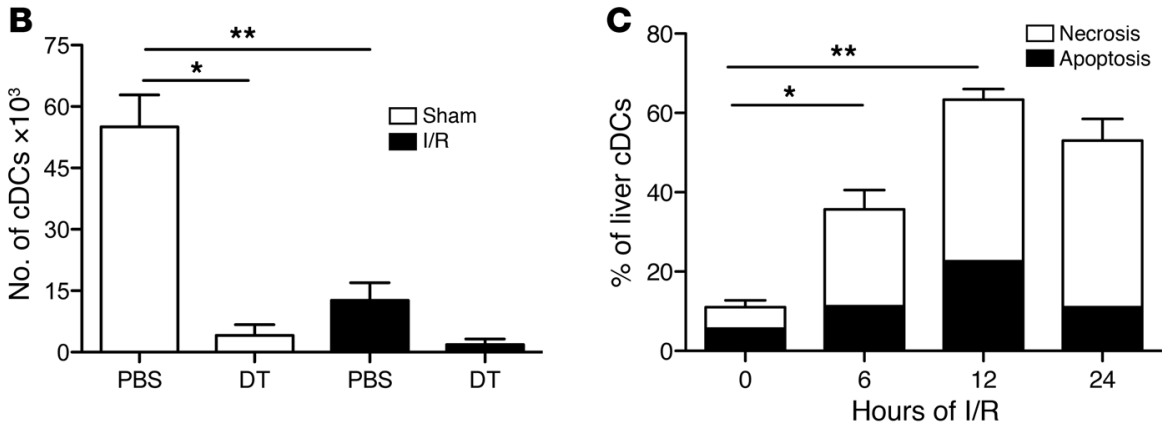

D
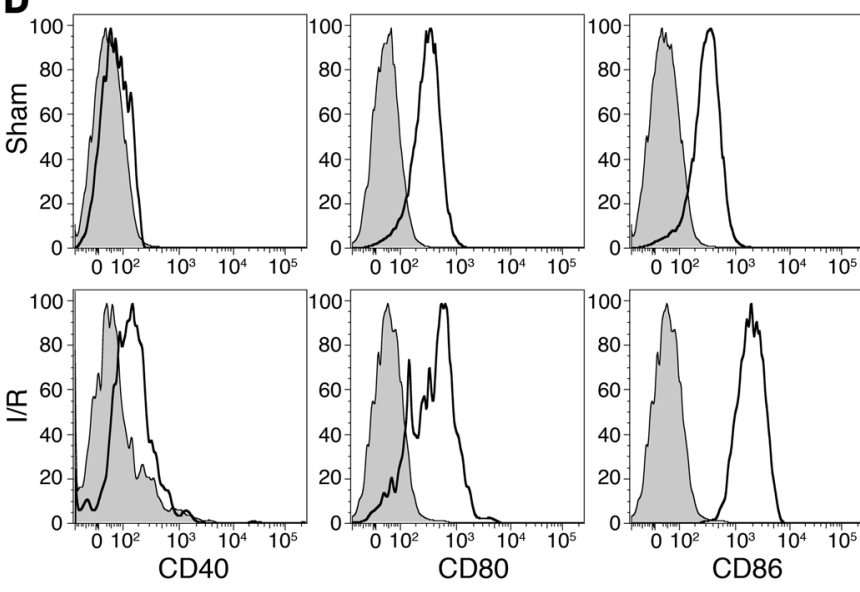

Figure 1

Liver $\mathrm{I} / \mathrm{R}$ induces $\mathrm{cDC}$ death. CD11c-DTR mice, which were pretreated with DT or PBS 18 hours earlier, underwent a sham laparotomy (Sham) or 1 hour of ischemia, followed by 12 hours of reperfusion (I/R). Ischemic liver cDCs were assessed by flow cytometry and are shown as (A) the percentage of hepatic leukocytes within each gated region or (B) the absolute number of cDCs within the ischemic liver. Ischemic liver cDCs from PBStreated CD11c-DTR mice were analyzed for (C) the percentage of apoptosis (Annexin $\mathrm{V}^{+} \mathrm{PI}^{-}$) and necrosis (Annexin $\mathrm{V}+\mathrm{Pl}+$ ) by flow cytometry or (D) CD40, CD80, and CD86 expression 12 hours after the sham procedure or I/R. Isotype controls are shown as shaded histograms. The bar graph represents CDC maturation data pooled from 3 independent experiments. Data represent mean \pm SEM (B-D). Data in all panels are representative of at least 3 independent experiments; $n=5$ mice per group. ${ }^{\star} P<0.05$; ${ }^{* *} P<0.01$. chymal cells (NPCs) from the ischemic lobes of PBS-treated CD11cDTR mice, subjected to 1 hour of ischemia and 12 hours of reperfusion. We chose to base our experiments on the 12-hour time point, as this is when maximal liver injury occurred (Supplemental Figure 1; supplemental material available online with this article; doi:10.1172/ JCI40008DS1). I/R resulted in a $65 \%$ reduction in the percentage and number of hepatic cDCs (Figure 1, A and B). As expected based on our prior experience (15), a single injection of diphtheria toxin (DT) depleted approximately $80 \%$ of liver cDCs in CD11c-DTR mice that underwent sham laparotomy. Meanwhile, in DT-treated mice, I/R resulted in almost complete loss of liver cDCs.

We performed serial analysis of the viability of liver cDCs to explain their disappearance in PBS-treated mice after I/R. We noted a time-dependent increase in liver $\mathrm{CDC}$ death that peaked at 12 hours after initiation of ischemia (Figure 1C). In line with earlier work by Loi et al. (16), we found that the remaining cDCs within the ischemic lobes were mature, with increased expression of CD40, CD80, and CD86 (Figure 1D). Similar maturation of cDCs occurred in the segments of the liver that were not subjected to ischemia (data not shown).

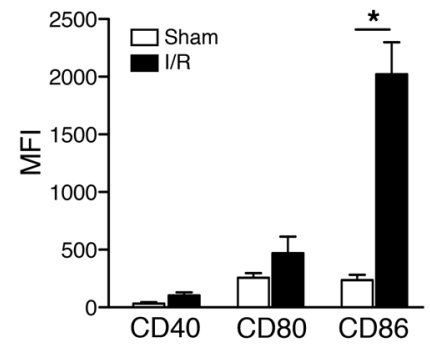

$c D C$ are protective in liver $I / R$. The functional importance of liver $\mathrm{cDCs}$ during $\mathrm{I} / \mathrm{R}$ is unknown. Therefore, we measured serum alanine aminotransferase (ALT) in DT- or PBS-treated mice subjected to a sham procedure or $\mathrm{I} / \mathrm{R}$. We found that $\mathrm{CDC}$ ablation markedly exacerbated liver injury following I/R (Figure 2A). To exclude the possibility that the increased liver injury in DT-treated mice resulted from nonspecific cell depletion or nonspecific immune priming, we reconstituted them with WT cDCs just prior to liver I/R. DT-treated mice injected i.v. with WT cDCs experienced similar liver injury to mice that underwent $\mathrm{I} / \mathrm{R}$ without $\mathrm{DC}$ ablation (Figure 2A). Further substantiating the protective role of cDCs, we found that adoptive transfer of WT cDCs into PBS-treated mice prior to I/R markedly reduced ALT levels. Histologic evaluation of the ischemic lobes after 12 hours of I/R confirmed the serum ALT data, as DT-treated mice displayed larger patches of hepatocyte necrosis than PBS-treated controls (Figure 2B).

Because proinflammatory cytokines and chemokines have been implicated as mediators of liver I/R injury (9), we next determined how cDC depletion altered the systemic immune response. DTtreated mice had significantly higher serum IL-6, MCP-1, and TNF 
A

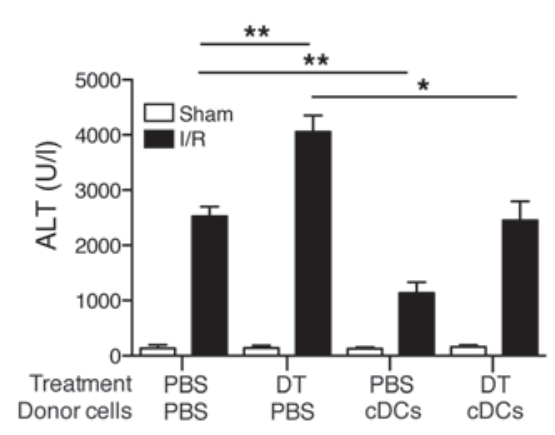

B
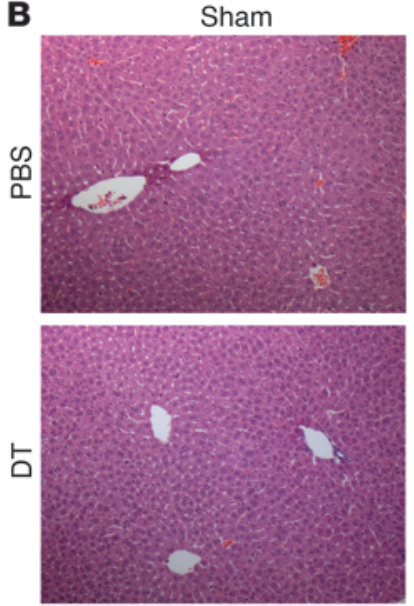
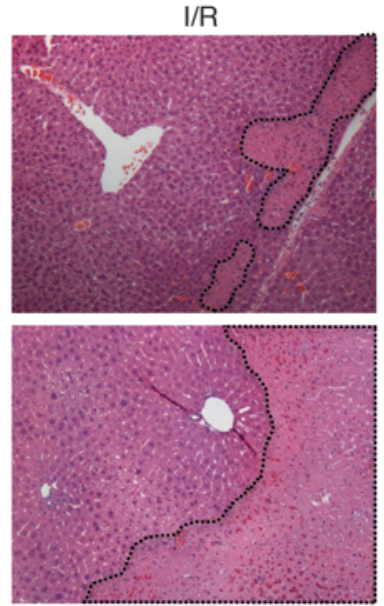

C
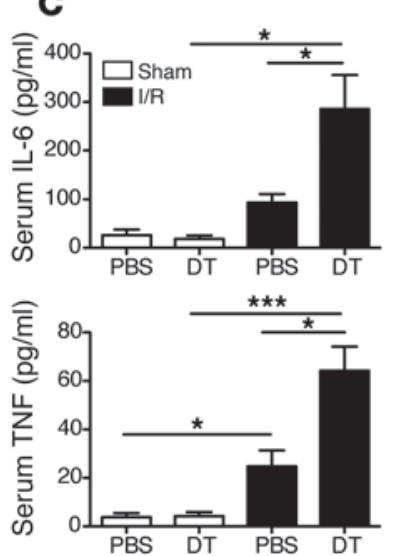
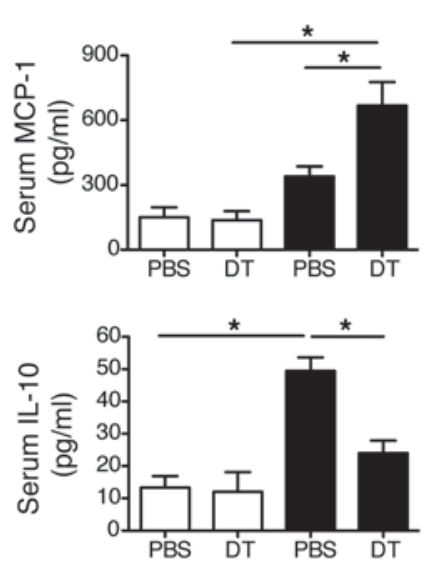

D
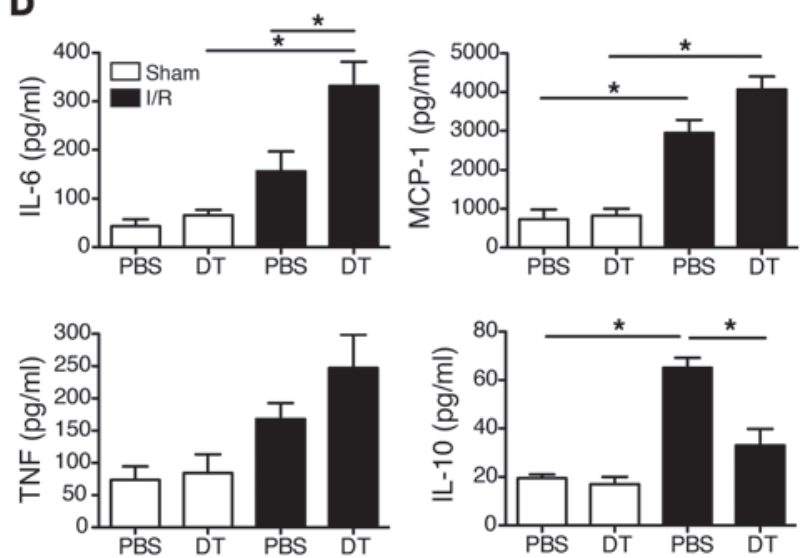

Figure 2

cDCs confer protection in I/R. CD11c-DTR mice were administered PBS or DT via i.p. injection. Twelve hours later, mice received an i.v. injection of $1 \times 10^{7}$ WT cDCs or PBS and then underwent sham laparotomy or I/R. (A) Serum ALT levels and (B) representative liver H\&E staining (original magnification, $\times 100$ ) are shown at 12 hours after sham procedure or liver l/R. Nonviable patches of ischemic lobes are demarcated by dashed lines. CD11C-DTR mice were pretreated with DT or PBS 12 hours earlier and then subjected to sham laparotomy or 12 hours of I/R. (C) Serum cytokines levels were measured or (D) ischemic liver CD45+ NPCs were harvested and cultured overnight in media prior to measurement of supernatant cytokines. Data represent mean \pm SEM $(\mathbf{A}, \mathbf{C}$, and $\mathbf{D})$. Data in all panels are representative of at least 3 independent experiments; $n=4-6$ mice per group. ${ }^{*} P<0.05 ;{ }^{* \star} P<0.01 ;{ }^{* \star \star} P<0.001$.

levels, but lower IL-10 levels 12 hours after I/R (Figure 2C). A similar pattern was observed in NPCs isolated from the ischemic lobes of DT-treated mice after overnight culture, when compared with ischemic NPCs from PBS-treated mice (Figure 2D).

IL-10 production by $c D C$ s confers protection from I/R injury. Because IL-10 is a potent antiinflammatory cytokine, we considered the reduction in systemic and local levels of IL-10 after depletion of cDCs as a possible mechanism to explain the increased liver injury in DT-treated mice. Hepatic DCs have been shown to exhibit tolerogenic properties and are thought to protect the liver from untoward inflammatory responses to foreign antigens entering the portal circulation (17). We recently discovered that freshly isolated human liver DCs exhibit a propensity for IL-10 secretion at baseline and after TLR stimulation (18). Because TLR9 has been implicated as an important regulator of inflammation through its detection of both bacterial and host DNA $(19,20)$, we sought to determine whether TLR9 activation and CDC production of IL-10 were necessary to confer protection against liver I/R injury. DT- treated mice were injected with PBS, $I l 10^{-/}$, $T l r 9^{-/-}$, or WT CDCs prior to the initiation of I/R. Measurement of serum ALT 12 hours later revealed that, unlike WT cDCs, $I l 10^{--}$and $\mathrm{Tl}^{-/-} \mathrm{cDCs}$ failed to confer protection (Figure 3A). There was similar reconstitution of adoptively transferred cDCs within the liver among the groups (Supplemental Figure 2). Moreover, the adoptive transfer of NK cells failed to impact liver I/R injury (data not shown). Mice that received $\mathrm{IllO}^{-/-}$or $\mathrm{Tl}^{-1-/}$ cDCs also had increased amounts of serum inflammatory cytokines (Figure 3B). To determine whether IL-10 production by CDCs in vivo depended on TLR9 activation, we injected WT (CD45.1) mice with congenic (CD45.2) Tlr9-/- or WT CDCs just prior to sham laparotomy or 12 hours of I/R. Intracellular cytokine analysis of the adoptively transferred cDCs isolated from sham and ischemic livers demonstrated that liver cDCs required TLR9 expression to produce IL-10 during I/R (Figure 3C). Taken together, these data suggest that TLR9 activation in cDCs induces IL-10 secretion that decreases the host response to danger signals released during sterile liver inflammation. 
A

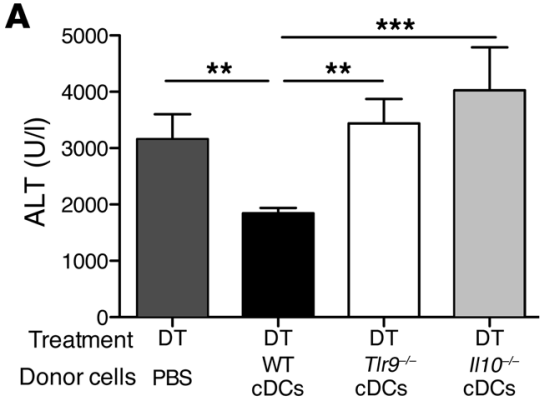

B

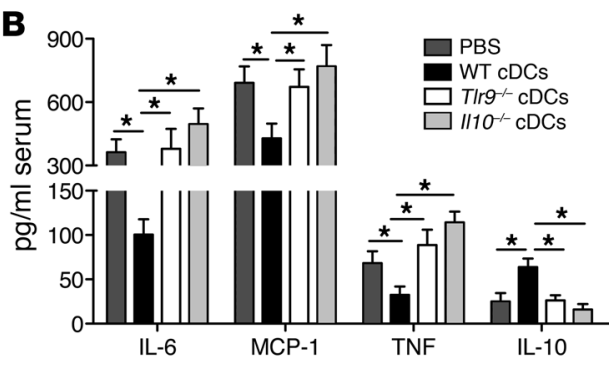

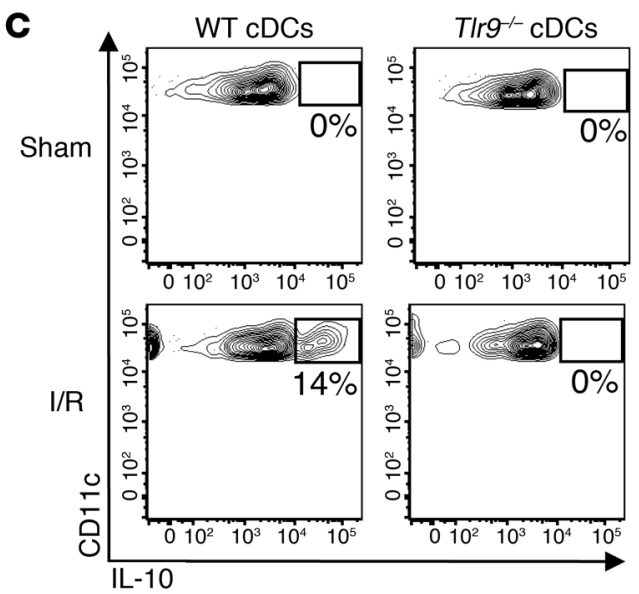

Figure 3

cDC-mediated protection in liver $\mathrm{I} / \mathrm{R}$ is TLR9 and IL-10 dependent. CD11C-DTR mice were administered DT via i.p. injection. Twelve hours later, mice received an i.v. injection of either PBS or $1 \times 10^{7} \mathrm{WT}$, TIr9-/-, or II10-/- cDCs just prior to I/R. (A) Serum ALT levels and (B) cytokines levels were measured 12 hours later. (C) WT or TIr9-/- (both CD45.2) cDCs were injected into WT (CD45.1) recipients just prior to sham laparotomy or I/R. Twelve hours later, ischemic liver CD45+ NPCs were isolated and cultured in media in the presence of Brefeldin A. Intracellular IL-10 production by $C D 45.2^{+} \mathrm{CD} 11 \mathrm{c}^{\text {hi }}$ hepatic $\mathrm{CDC}$ s was assayed 6 hours later and is expressed as the percentage within each gated region based on IL-10 isotype control antibody staining. The bar graph represents pooled data from 3 independent experiments. Data represent mean \pm SEM and are representative of at least 2 independent experiments; $n=4-6$ mice per group. ${ }^{\star} P<0.05$; ${ }^{\star \star} P<0.01 ;{ }^{* \star} P<0.001$
Host DNA induces liver cDC IL-10 production through TLR9. It is becoming increasingly apparent that under certain conditions, endosomal TLRs can be activated by self nucleic acids $(21,22)$. Since liver I/R causes hepatocyte death, we hypothesized that the release of endogenous DNA was responsible for the induction of IL-10 secretion by WT cDCs. WT and Tlr $9^{-/-}$cDCs were cultured in media with or without supernatant from necrotic WT hepatocytes (conditioned media) for 18 hours prior to measurement of supernatant IL-10. WT cDCs secreted considerably more IL-10 than Tlr9-/- cDCs in conditioned media (Figure 4A). The addition of DNAse confirmed that DNA was a major contributor to IL-10 secretion by WT cDCs. Intracellular DNA recognition occurs via TLR9 and also has been recently attributed to the pyrin and HIN domain-containing protein family member called absent in melanoma 2 (AIM2) (23). Using an inhibitory CpG (iCpG) sequence, which blocks TLR9 activation, we found that the $\mathrm{CDC}$ response to conditioned media largely depended on TLR9 (Figure 4A). Although TLR3 was recently recognized as an endogenous sensor of tissue necrosis (4), we found minimal changes in cytokine production by $\mathrm{cDCs}$, cultured with conditioned media pretreated with RNAse (data not shown).

The liver is enriched in a vast array of immune cells that interact with each other and modulate the organ's overall response to infection and inflammation. To determine whether our data on isolated liver $\mathrm{cDCs}$ were relevant in the context of the global hepatic immune repertoire, we incubated NPCs overnight in conditioned media. In some cases we depleted native cDCs (NPCs$\mathrm{cDCs}$ ) or replaced them with transgenic cDCs. Despite comprising only $4 \%$ of liver leukocytes (Figure $1 \mathrm{~A}$ ), cDCs were responsible for more than one-third of IL-10 production by NPCs in response to conditioned media (Figure 4B). Only the addition of WT cDCs, and not $\mathrm{Il}^{-10^{--}}$or $\mathrm{Tl} \mathrm{r}^{-/-} \mathrm{CDCs}$, to NPCs depleted of NPCs-cDCs restored IL-10 production. Consistent with our data from experiments in which we used isolated cDCs, we found that bulk NPCs produced substantially less IL-10 when cultured with conditioned media that contained either DNAse or iCpG. Collectively, these data support the notion that liver $\mathrm{CDCs}$ contribute to the overall IL-10 response of NPCs via TLR9-mediated activation by endogenous hepatocyte DNA.

Inflammatory monocytes are recruited to the ischemic liver and worsen $I / R$ injury. ROS generation has been shown to play an important role in potentiating liver I/R injury (24). We therefore investigated whether hepatic cDCs could modulate the oxidative capacity of immune cells during I/R and thereby limit liver injury. We chose to focus on neutrophils, Kupffer cells, and inflammatory monocytes, as they have all been implicated as major producers of ROS during inflammation and infection $(24,25)$. DT treatment followed by 12 hours of I/R increased ROS production in inflammatory monocytes (CD11b int $L y 6 C^{\text {hi }}$ ) to a much greater extent than in neutrophils $\left(\mathrm{CD} 11 \mathrm{~b}^{\mathrm{hi}} \mathrm{Ly} 6 \mathrm{G}^{+}\right)$and Kupffer cells (CD11b ${ }^{\text {lo }}$ 4 $/ 80^{+}$) (Figure 5A).

The contribution of inflammatory monocytes to hepatic I/R injury remains poorly defined. To evaluate the impact of the increased oxidative burst in monocytes that accompanied cDC ablation, we first examined inflammatory monocyte recruitment to the liver during I/R. We noted a moderate increase in the percentage (Figure $5 \mathrm{~B}$ ) and absolute number (data not shown) of inflammatory monocytes within the ischemic lobes of the liver following I/R that was associated with a concomitant decrease in their presence within the bone marrow. Next, we performed a series of in vivo antibodymediated depletion experiments to determine the relative importance of inflammatory monocytes in liver I/R injury. WT mice were pretreated with anti-Gr1 to deplete both Ly6G- and Ly6Chi-expressing cells. In parallel, a second group of mice were injected with the 
A

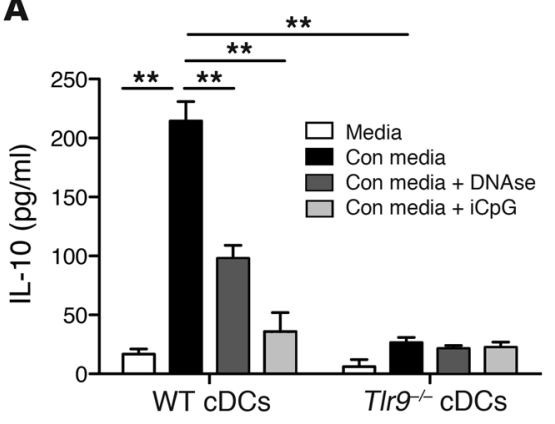

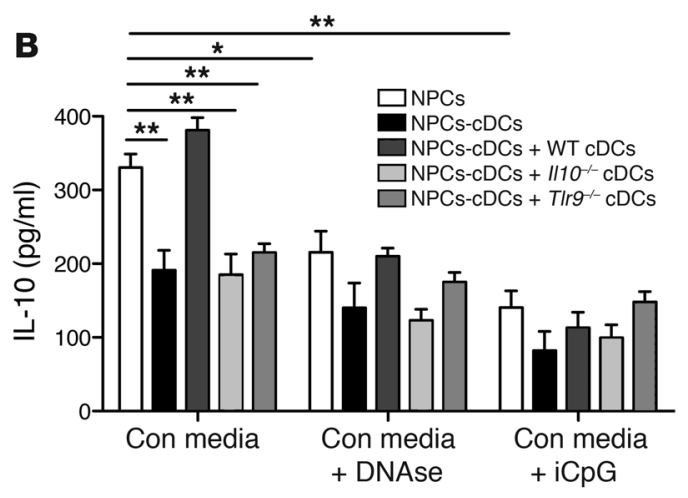

Figure 4

Endogenous DNA promotes cDC IL-10 production through TLR9. (A) Immunomagnetic bead-purified cDCs from WT or TIr9--- mice were cultured in media alone or with conditioned media (Con media) from necrotic hepatocytes. Some wells containing conditioned media were pretreated with DNAse I prior to coculture with cDCs, while iCpG was added to other wells. Supernatant IL-10 was measured 18 hours later using a cytometric bead array. (B) Liver CD45+ NPCs were isolated from WT mice and cultured in conditioned media, with or without DNAse I pretreatment or iCpG, as in A. In selected wells, NPCs were depleted of CDCs prior to the addition of conditioned media. WT, TIr9-/-, or $1110^{-/-}$cDCs were added back to certain wells containing NPCs-cDCs. Supernatant IL-10 was measured 18 hours later. Data depict mean \pm SEM from triplicate wells and are representative of 3 independent experiments. ${ }^{\star} P<0.05 ;{ }^{* \star} P<0.01$.

monoclonal antibody $1 \mathrm{~A} 8$ that depletes Ly6G-expressing neutrophils exclusively (26). We observed a significant decrease in ALT after I/R in mice treated with either antibody (Figure 5C). Since both antibodies eliminated intrahepatic neutrophils (Figure 5D), the lower level of liver injury after anti-Gr1 treatment appeared to result from the additional depletion of inflammatory monocytes.

In addition to CD11b and Ly6C, inflammatory monocytes are characterized by expression of CCR2 (27). Mice lacking CCR2 are more susceptible to infection but, conversely, are more resistant to inflammatory conditions such as atherosclerosis and autoimmune encephalitis $(28,29)$. To confirm that inflammatory monocytes contribute to liver injury, we performed I/R in Ccr $2^{-/-}$mice. When compared with WT controls, $\mathrm{Cr} 2^{-/-}$mice undergoing 12 hours of $\mathrm{I} / \mathrm{R}$ had virtually no inflammatory monocytes within their ischemic lobes but contained a relative abundance of CD11 ${ }^{\text {int }}$ Ly $6 \mathrm{C}^{\text {hi }}$ cells within their bone marrow (Figure 6A). There were no differences in the number of neutrophils or Kupffer cells within the ischemic livers of WT and $\mathrm{Ccr} 2^{-/-}$mice after I/R (data not shown). These results, coupled to the fact that $\mathrm{Ccr} 2^{-/-}$mice were protected from hepatic I/R (Figure 6B), suggest that inflammatory monocytes aggravate liver injury via CCR2-mediated recruitment to the ischemic liver.

cDC $I L-10$ inhibits inflammatory monocyte function during $I / R$. To further characterize the effect of $c D C$ s during I/R, we examined changes in the production of cytokines by inflammatory monocytes. Intracellular cytokine analysis of ischemic lobe NPCs revealed that DT treatment prior to I/R increased the production of TNF and IL-6 by inflammatory monocytes (Figure 7A). In line with our data on oxidative burst (Figure 5A), cDC ablation in I/R exerted its greatest effect on inflammatory monocytes at the same time as minimal changes in cytokine production were observed in neutrophils and Kupffer cells (data not shown).

To ascertain whether IL-10 production by cDCs accounted for the suppression of inflammatory monocyte function, we first examined changes in IL-10 receptor expression after I/R. IL-10 receptor expression was significantly increased on inflammatory monocytes but not neutrophils and Kupffer cells within the ischemic liver (Figure 7B). Next, we adoptively transferred WT or $I l 10^{-/-}$ cDCs into DT-treated CD11c-DTR mice. Mice were then subjected to 12 hours of I/R before levels of serum ALT, inflammatory monocyte ROS, and cytokine production were determined. Although mice injected with $\mathrm{IllO}^{-/-} \mathrm{cDCs}$ experienced higher ALT levels than those injected with WT cDCs (Figure $3 \mathrm{~A})$, the number of inflammatory monocytes and neutrophils recruited to the ischemic livers was similar between the groups (data not shown). Inflammatory monocytes from mice injected with $I l 10^{-/} \mathrm{cDCs}$ produced more ROS, IL- 6 , and TNF compared with mice receiving WT cDCs (Figure 7, C and D). Collectively, these data suggest that IL-10 secretion by cDCs confers protection by suppressing inflammatory monocyte function during hepatic I/R.

\section{Discussion}

The host faces a myriad of signals released during tissue injury (30). Liver I/R induces a combination of necrosis and apoptosis (31). After uptake of apoptotic cells, cDCs have been shown to secrete lower amounts of proinflammatory cytokines (32) and produce more IL-10 (33). In contrast, endogenous ligands released by necrotic cells evoke proinflammatory responses in DCs (34). How innate immune cells integrate these often opposing signals and the mechanisms that regulate the ensuing immune response are unclear. Our data suggest that during liver I/R, the net effect of these contrasting signals on cDCs is governed by TLR9. This is exemplified by increased I/R injury in DT-treated CD11c-DTR mice injected with $\operatorname{Tlr}^{-1-} \mathrm{cDCs}$ compared with animals receiving WT cDCs. Moreover, we show that the antiinflammatory response evoked in cDCs is dependent on endogenous DNA and serves as a natural regulatory pathway that is activated to increase hepatic resistance to I/R. A schematic outlining the mechanism revealed by our experiments is shown in Figure 8.

The importance of TLR9 in various models of monomicrobial infection has been well established $(35,36)$. Although the precise mechanism of protection appears to depend on the infectious model, the principle by which TLR9 activation confers resistance to foreign pathogens is essentially the same among models of infection. Bacterial DNA engages host cell TLR9, inducing an proinflammatory immune response that curtails the spread of infection and ultimately eliminates the invading organism. Thus, the host has the capacity to respond to infection and trauma in entirely different ways despite the common involvement of TLR9. While teleologically this makes sense, as the outcome of TLR9 activation in both settings serves to limit host injury, how the immune system is able to distinguish between threats from pathogens and endogenous danger signals following trauma remains unclear. One 

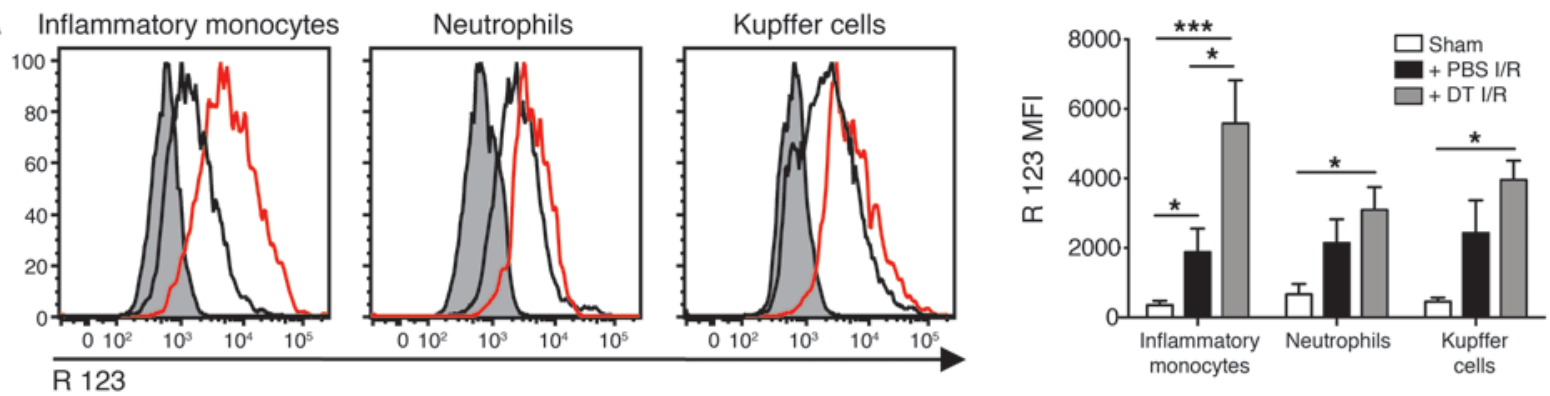

B
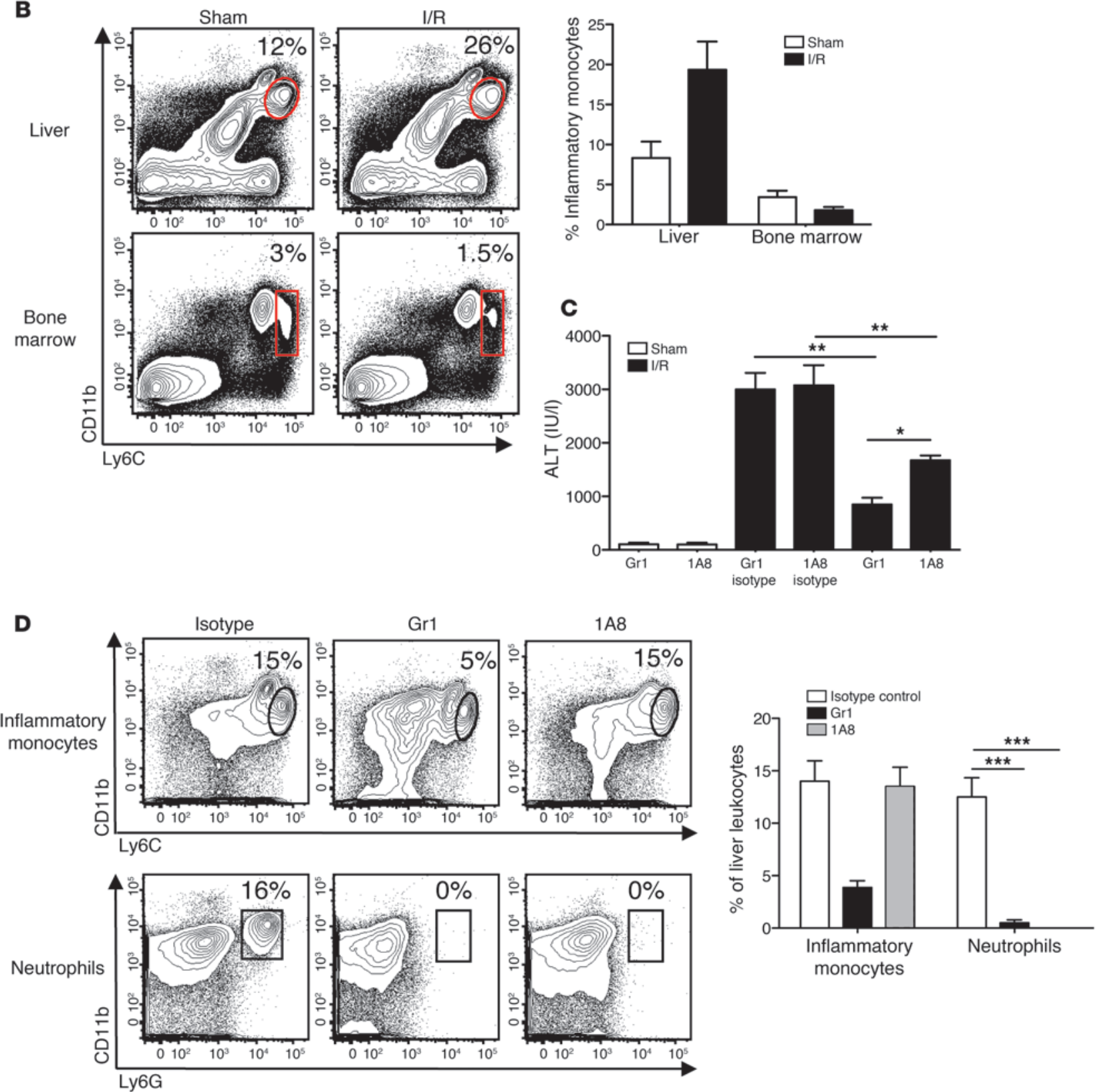


\section{Figure 5}

Inflammatory monocytes exacerbate liver I/R injury. CD45+ NPCs were isolated from the ischemic livers of CD11c-DTR mice after PBS or DT pretreatment and 12 hours of I/R or sham procedure. (A) Histograms depict inflammatory monocyte (CD11 bintLy6Chi), neutrophil $\left(\mathrm{CD} 11 \mathrm{~b}^{\text {hi }} \mathrm{Ly}_{6 \mathrm{G}}{ }^{+}\right)$, and Kupffer cell $\left(\mathrm{CD} 11 \mathrm{~b}^{\mathrm{lo}} \mathrm{F} 4 / 80^{+}\right)$ROS production, based on the conversion of dihydrorhodamine 123 to its oxidized form, rhodamine (R) 123 (shaded areas indicate sham procedure; black lines indicate PBS and I/R treatment; red lines indicate DT and I/R treatment). The bar graph represents ROS data pooled from 3 experiments. (B) Inflammatory monocyte frequencies within the ischemic liver and bone marrow of WT mice after 12 hours of I/R or sham surgery are represented as a percentage of $\mathrm{CD} 45^{+}$hepatic leukocytes or of live bone marrow cells. The bar graph represents pooled data from 3 experiments. (C and D) WT mice received anti-Gr1, 1A8, or isotype controls, 24 and 2 hours before I/R or sham procedure. Percentages of cells within selected gates are indicated. (C) Twelve hours later, serum ALT levels and (D) inflammatory monocyte and neutrophil composition within the ischemic liver were determined. Percentages of cells within selected gates are indicated. The bar graph represents pooled data from 3 independent experiments. Data represent mean \pm SEM. ${ }^{\star} P<0.05 ;{ }^{* *} P<0.01 ;{ }^{* *} P<0.001$.

possible explanation is that because of differences in their conformation, bacterial and endogenous TLR9 ligands trigger distinct downstream signaling pathways and transcriptional profiles (37). In fact, both TLR2 and TLR4 have been shown to respond differentially to their respective endogenous and pathogenic ligands, although the mechanisms have yet to be defined $(38,39)$. However, another possibility is that TLR9 ligands activate other receptors that modulate the TLR9 signaling pathway. Indeed, after acetaminophen-induced liver necrosis, activation of CD24 by HMGB1 and heat shock proteins negatively regulated their inflammatory activity by suppressing NF- $\mathrm{KB}$ activation (34). When compared with WT mice, CD24-deficient animals were more susceptible to pathologic cell death induced by acetaminophen but were equally responsive to pathogen-associated molecular patterns, such as LPS.

We provide functional evidence supporting the protective role of $\mathrm{cDCs}$ in I/R via their production of IL-10. In congruence with our findings, reports by others using recombinant IL-10 or neutralizing IL-10 antibodies showed that exogenous as well as endogenous IL-10 increased hepatic resistance to I/R injury (40-42). The source and cellular mechanisms accounting for IL-10-mediated protection in these studies were not determined. Although IL-10 appeared to be the critical cytokine in modulating the effect of cDCs on I/R injury, both WT and Tlr9--- DCs are capable of producing proinflammatory cytokines in response to danger signals such as $\operatorname{HMGB1}(43,44)$. We found that the levels of IL-6 and TNF production by WT, $\mathrm{Tlr}^{-/-}$, and $I l 10^{-/-} \mathrm{cDCs}$, in response to conditioned media or stimulation with recombinant HMGB1 in vitro, were comparable (Supplemental Figure 3). Furthermore, there were no significant differences in IL-10 production between WT and Tlr $9^{-/-} \mathrm{cDCs}$ after stimulation with recombinant HMGB1 (data not shown). These findings imply that either a critical amount of IL-10 in vivo needs to be reached before significant liver protection is attained or the balance between antiinflammatory and proinflammatory cytokines determines the degree of I/R injury. Various other models of liver injury support the notion that an optimal IL-10 to proinflammatory cytokine ratio is required to limit tissue damage $(45,46)$.

In contrast to our data, an earlier report by Tsung et al. suggests that DCs promote I/R injury through a TLR4-dependent pathway involving HMGB1 (47). We believe that this discrepancy on the role of DCs in liver I/R stems from differences between the models used. While we investigated cDC function under steady-state conditions specifically, Tsung and colleagues treated mice with GM-CSF for several days prior to performing I/R. We have shown that use of GM-CSF to expand hepatic DCs in vivo can profoundly influence their recruitment, development, and function $(48,49)$. In addition, the effects of GM-CSF on increasing macrophage number and function (50) as well as our emphasis on studying CD $11 c^{\text {hi }} \mathrm{cDCs}$, as opposed to bulk CD $11 \mathrm{c}^{+} \mathrm{DCs}$, may also account for some of the observed differences.

The recruitment of neutrophils and monocytes to sites of infection or tissue injury is a cardinal feature of inflammation. While the role of neutrophils in $\mathrm{I} / \mathrm{R}$ has been established, the contribution of inflammatory monocytes to liver I/R injury has been poorly defined. Our results indicate that CCR2-mediated signaling is critical for the recruitment of inflammatory monocytes from the bone marrow to the ischemic liver. This is in line with published data linking CCR2 with egress of $\mathrm{CD} 11 \mathrm{~b}^{+} \mathrm{Ly} 6 \mathrm{C}^{\text {hi }}$ monocytes from the bone marrow to sites of infection (51). Whether CCR2 is also involved in monocyte migration from the liver sinusoids into the hepatic parenchyma is unknown. Our findings that we believe to be novel on the detrimental role of inflammatory monocytes in liver I/R and their dependence on CCR 2 are consistent with results from other models of $\mathrm{I} / \mathrm{R}$ involving the kidney and brain $(52,53)$. In addition, our results reveal that the capacity of $\mathrm{cDCs}$ to limit $\mathrm{I} / \mathrm{R}$ injury depends on the suppressive effects of IL-10 on inflammatory monocytes specifically. Ablation of cDCs in CD11c-DTR mice prior to I/R increased inflammatory monocyte function but failed to alter cytokine and ROS production by Kupffer cells and neutrophils. While IL-10 appeared to be a critical component of the regulatory effect of $\mathrm{cDCs}$, our in vitro data suggest that other hepatic NPCs are also capable of producing IL-10. The protective effect of cDCs may therefore be related to additional factors such as their location within the liver or the kinetics of

\section{Figure 6}

Inflammatory monocytes depend on CCR2 to promote liver I/R injury. WT and Ccr2-/mice underwent 12 hours of $\mathrm{I} / \mathrm{R}$ or sham laparotomy, at which time $(\mathbf{A})$ the number of inflammatory monocytes within the ischemic liver and bone marrow (i.e., per femur) and (B) serum ALT levels were determined. Data represent mean \pm SEM and are representative of at least 2 independent experiments; $n=4-6$ mice per group. ${ }^{*} P<0.05$; ${ }^{\star \star} P<0.01 ;{ }^{* \star} P<0.001$.
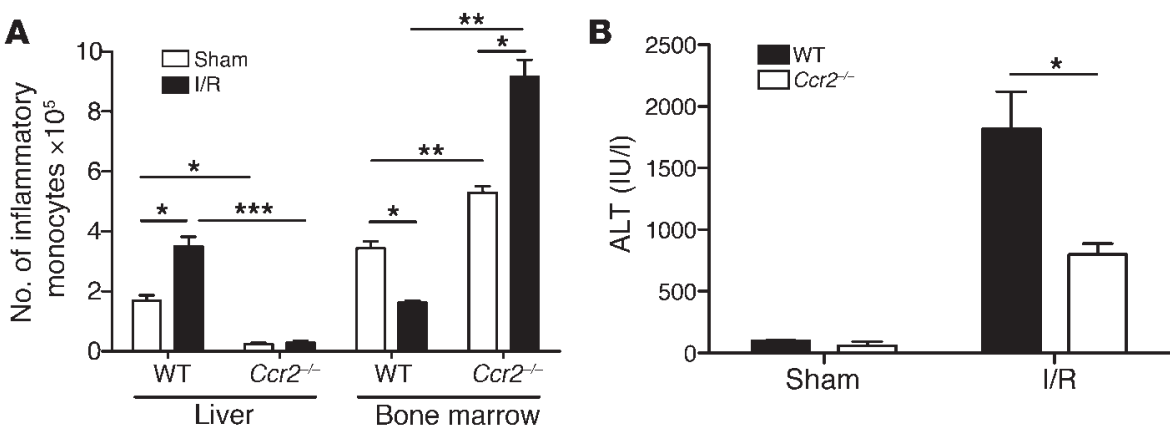

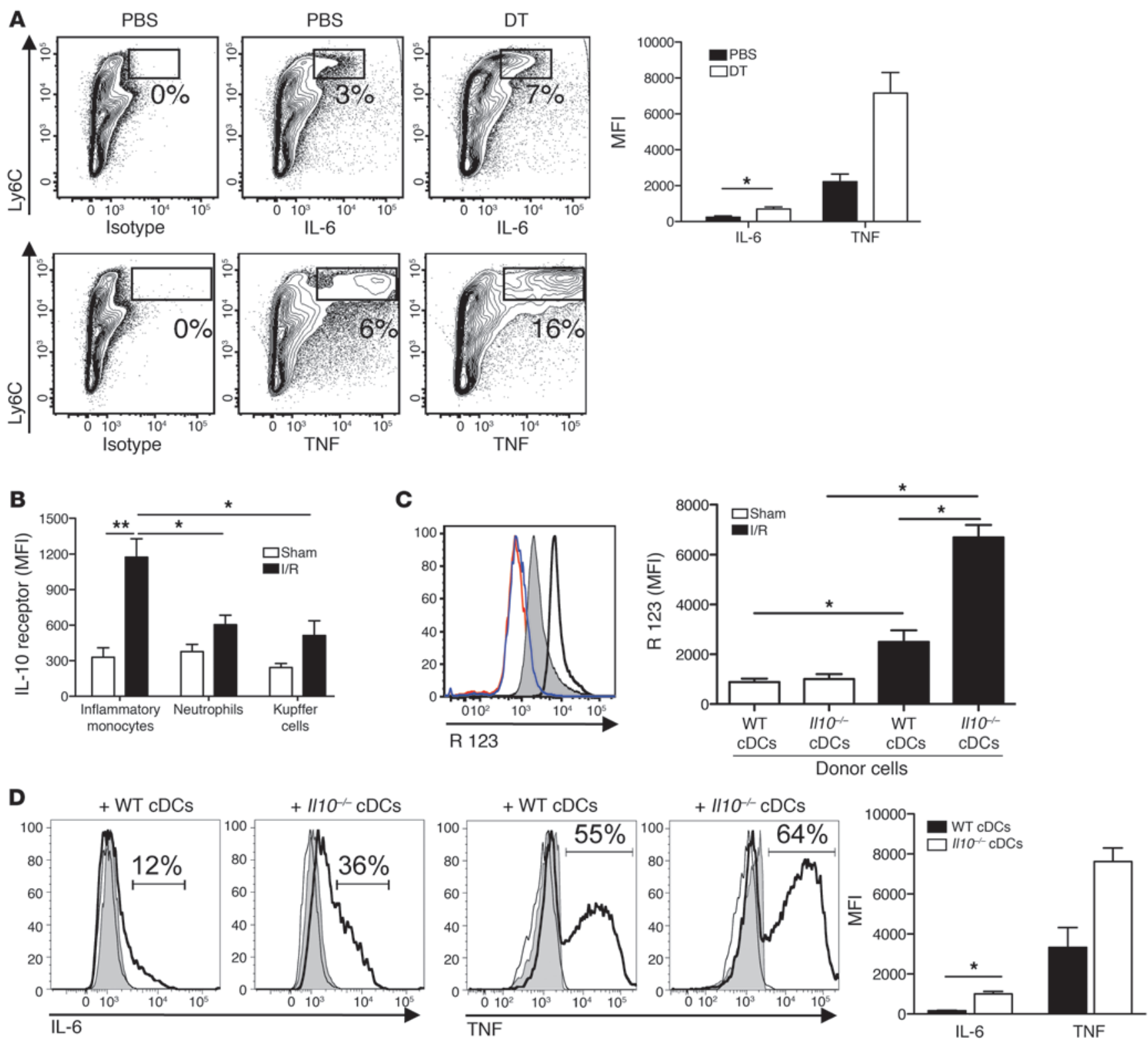

Figure 7

CDC IL-10 suppresses inflammatory monocyte function during I/R. Ischemic liver CD45+ NPCs from CD11C-DTR mice treated with DT or PBS prior to 12 hours of I/R were cultured with Brefeldin A. (A) Intracellular IL-6 and TNF was determined 6 hours later. Numbers indicate the percentages within each gated region of cytokine staining in excess of isotype controls. More than $95 \%$ of the Ly6Chi cells depicted are CD $11 \mathrm{~b}^{+}(\mathrm{data}$ not shown). The bar graph depicts inflammatory monocyte cytokine production as MFI above isotype pooled from 3 experiments. (B) IL-10 receptor expression was measured on inflammatory monocytes, neutrophils, and Kupffer cells from ischemic livers of PBS-treated CD11c-DTR mice 12 hours after I/R or sham procedure. MFI values above isotype controls are shown and pooled from 3 experiments. Recipient CD11c-DTR mice pretreated with DT were injected with WT or $1 / 10^{-/-} \mathrm{cDC}$ just prior to hepatic ischemia or sham procedure. (C) Twelve hours later, oxidative burst was measured (red line indicates sham treatment with WT cDCs; blue line indicates sham treatment with $/ / 10^{-/-} \mathrm{cDCs}$; shaded area indicates I/R treatment with WT cDCs; bold line indicates I/R treatment with $/ 110^{-/-} \mathrm{CDCs}$ ). (D) Intracellular IL-6 and TNF production by inflammatory monocytes from liver CD45+ NPCs was measured and expressed as a percentage above isotype controls (black lines indicate isotype control; shaded area indicates sham treatment; bold lines indicate I/R treatment). The bar graphs in $\mathbf{C}$ and $\mathbf{D}$ depict data pooled from 3 experiments. (D) MFI values above isotype controls are shown only for l/R mice. Data represent mean \pm SEM and are representative of at least 2 experiments; $n=4-6$ mice per group. ${ }^{\star} P<0.05 ;{ }^{\star \star} P<0.01$.

cDC IL-10 production during I/R. In addition to IL-10, cDCs may restrict inflammatory monocyte function via additional pathways. Alternate mechanisms such as activation of antiinflammatory macrophages by apoptotic $\mathrm{CDCs}$ during I/R are also possible.
It is worth mentioning that substantial strides have been made recently in understanding the role of other intracellular pattern recognition receptors in the context of inflammation. In particular, the nucleotide-binding domain leucine-rich repeat con- 


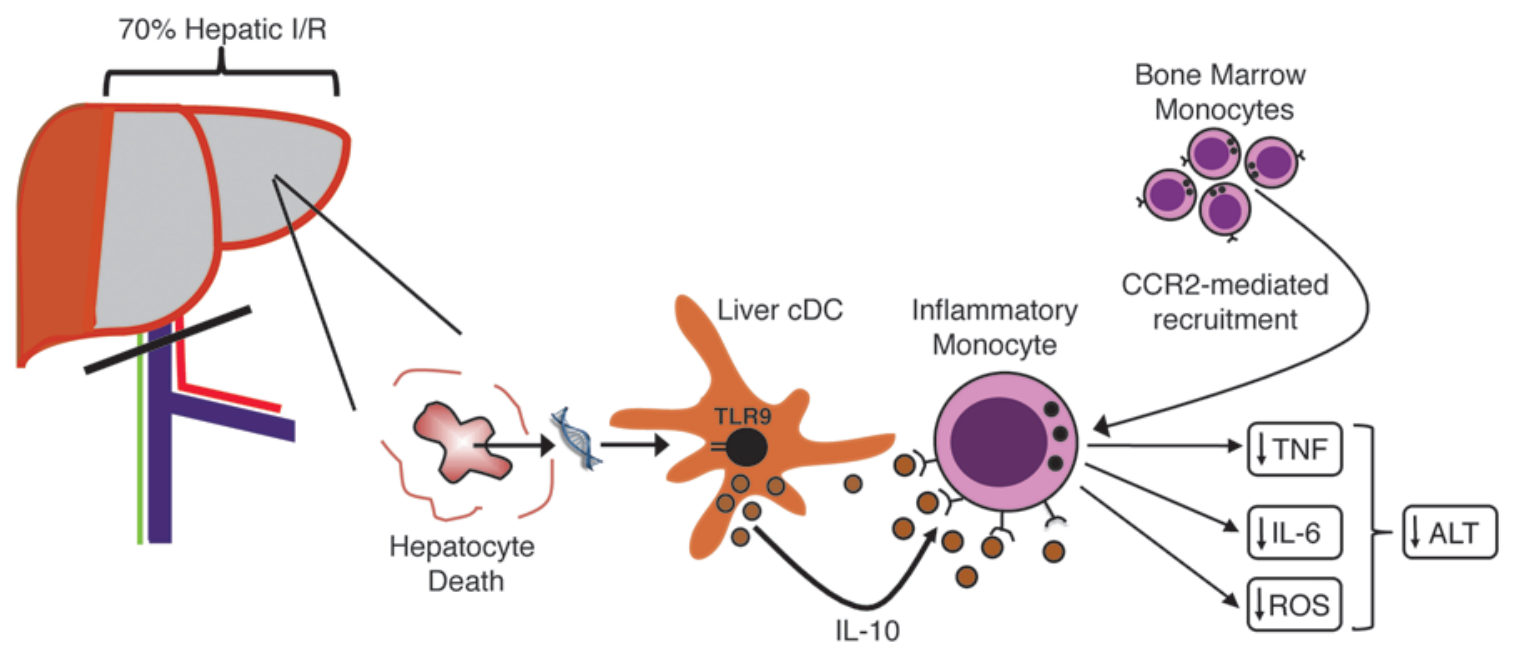

Figure 8

Role of cDCs in hepatic I/R. Vascular occlusion involving $70 \%$ of the liver for 1 hour followed by 12 hours of reperfusion induces hepatocyte death. The release of host DNA activates hepatic CDCs through TLR9. The ensuing production of IL-10 by cDCs causes suppression of inflammatory monocytes that are recruited to the ischemic liver via CCR2. Reduced production of IL-6, TNF, and ROS by inflammatory monocytes lowers ALT levels and reduces liver injury.

taining receptors (NLRs), which form part of the inflammasome protein complex, have been shown to play an important role in a number of models of infection and inflammation (54). It is likely that inflammasomes contribute to liver I/R injury, as the requisites for their activation, such as ROS and various DAMPs, including DNA, are released during hepatic ischemia $(55,56)$. In addition, IL-1 $\beta$ and IL-18, which are secreted following inflammasome activation, have been implicated as promoters of hepatic $\mathrm{I} / \mathrm{R}$ injury $(57,58)$. The precise interplay between TLRs and inflammasome pathways in DCs during sterile inflammation is unknown and warrants investigation.

This study does not rule out the possibility that other tissue-resident DCs exhibit similar properties. In fact, our adoptive transfer experiments highlight the protective benefit of spleen-derived $\mathrm{cDCs}$ in hepatic I/R. While the use of freshly isolated liver cDCs would have been ideal, low cell yield made this impractical. However, the observation that resident renal DCs potentiate kidney $\mathrm{I} / \mathrm{R}$ injury (59) suggests that our findings on the protective role of cDCs might be liver specific. Tissue-resident DCs may undergo local conditioning and adapt their function to the surrounding environment. This notion is supported by data on the unique regulatory mechanisms exhibited by intestinal DCs (60) and our prior work highlighting functional differences between freshly isolated human liver and autologous blood DCs (18). Another limitation of our study is that cDC depletion in the CD11c-DTR mouse is not confined to the liver. Consequently, we did not prove that the protective effects of $\mathrm{cDCs}$ during hepatic I/R are solely attributable to intrahepatic cDCs. Furthermore, after adoptive transfer $\mathrm{cDCs}$ accumulated in other organs besides the liver (data not shown), and thus we cannot definitively conclude that their effects were specific to the liver.

In addition to liver dysfunction, severe hepatic I/R injury induces a systemic inflammatory response that can result in multiple organ failure and death. By identifying $\mathrm{CDCs}$ as an important negative regulator of the inflammatory response in liver $I / R$, we provide evidence for what we believe to be a novel mechanism, by which the innate immune system can limit untoward tissue injury. Harnessing the antiinflammatory potential of cDCs via TLR9 may represent a novel approach to limiting I/R injury. Improved understanding of the complex interactions between DAMPs, pathogenassociated molecular patterns, and pattern recognition receptors on immune cells will promote the development of novel, more effective strategies to limit liver I/R injury.

\section{Methods}

Animals and procedures. CD45.2 C57BL/6 (B6), Il10-/-, and WT CD45.1 mice (10-18 weeks) were purchased from The Jackson Laboratory. $\mathrm{Tlr}^{-/-}$(CD45.2; obtained from S. Akira, Osaka University, Osaka, Japan) and CD11c-DTR (The Jackson Laboratory) mice on a B6 background were bred at MSKCC. $\mathrm{Ccr} 2^{-/-}$mice on a B6 background were a gift from E. Pamer (Sloan-Kettering Institute, New York, New York, USA). For systemic cDC depletion, CD11cDTR mice were injected i.p. with 4 ng DT per gram of body weight (D-0564; Sigma-Aldrich) 12-18 hour prior to I/R. In line with our prior experience, administration of DT did not deplete Kupffer cells (CD11 $\left.\mathrm{b}^{\text {int }} \mathrm{F} / 80^{\text {hi }}\right)$ and hepatic CD11 $c^{\text {int }}$ cells that included plasmacytoid DCs $\left(C D 11 c^{\text {int }}\right.$ PDCA $\left.^{+}\right)$ (15). Depletion of Ly6Chi- and Ly6G-expressing cells was accomplished with i.p. injections of $500 \mu \mathrm{g}$ anti-Gr1 antibody (RB6-8C5; Monoclonal Core Facility, Sloan-Kettering Institute) or isotype control (RatIgG2b; Monoclonal Core Facility, Sloan-Kettering Institute) 24 and 2 hours before I/R. Selective depletion of neutrophils was carried out with the same dosing regimen, using an Ly6G-specific antibody (1A8; BioXCell) or isotype control (RatIgG2a; BioXCell). In vivo expansion of DCs was accomplished with daily i.p. injections of $10 \mu \mathrm{g}$ of recombinant human fms-like tyrosine kinase 3 ligand (Flt3L; Amgen) for 10 days. Blood was obtained by direct cardiac puncture for isolation of serum. All animals were maintained in a pathogenfree animal housing facility at MSKCC. All procedures were approved by the MSKCC Institutional Animal Care and Use Committee.

Model of liver I/R. A model of segmental (70\%) warm hepatic ischemia was used, which is similar to that described previously (61). Briefly, under ketamine $(1 \mathrm{mg} / \mathrm{ml})$ and xylazine $(1 \mathrm{mg} / \mathrm{ml})$ anesthesia, an upper midline abdominal incision was made. The liver was exteriorized, and the hilum was exposed. The vasculature supplying the left and median lobes (ischemic 
lobes) of the liver was occluded with a microvascular clamp (Roboz Surgical Instruments) for 60 minutes. Evidence of ischemia during the clamping period was confirmed by blanching of the ischemic lobes. Reperfusion was initiated by removal of the clamp, and gross evidence of reperfusion was confirmed by color change of the ischemic lobes. Sham mice underwent the same procedure without the application of a vascular clamp. All mice received $200 \mu \mathrm{l}$ of sterile saline i.p. just prior to abdominal closure. The temperature during the procedure was maintained with the use of a warming pad and heating lamp. At the end of the observation period, mice were euthanized by carbon dioxide inhalation.

Assessment of liver damage. Hepatic injury after I/R was determined by measuring serum ALT levels, using the Olympus AU400 Chemistry Analyzer (Laboratory of Comparative Pathology, Sloan-Kettering Institute). Ischemic lobes were removed from mice immediately after sacrifice and fixed in $10 \%$ formalin. Samples were embedded in paraffin and cut to $5-\mu \mathrm{m}$-thick sections. Tissues were then stained with H\&E, and the slides were assessed for inflammation and tissue damage, using an Axioplan 2 wide-field microscope (Zeiss).

Cell isolation and adoptive transfer. Liver NPCs were isolated as previously described, with minor modifications (62). After the liver was harvested and processed, the supernatant was centrifuged ( $450 \mathrm{~g}$ for 5 minutes) to isolate NPCs. The NPCs were further enriched by a $40 \%$ (w/v) Optiprep (Sigma-Aldrich) density gradient per the manufacturer's protocol. The layer of low-density cells at the interface was harvested to obtain NPCs. $\mathrm{CD} 45^{+}$hematopoietic cells were isolated from NPCs by positive selection, using immunomagnetic beads as per the manufacturer's instructions (Miltenyi Biotec). WT hepatocytes were separated from NPCs following in situ perfusion with collagenase (type IV, $1 \mathrm{mg} / \mathrm{ml}$; Sigma-Aldrich) and gentle mechanical disruption of liver tissue. This was followed by 5 cycles of centrifugation ( $50 \mathrm{~g}$ for 2 minutes), in which the pellet, containing hepatocytes, was separated from the supernatant. Hepatocyte purity exceeded $90 \%$, as assessed by light microscopy. Bone marrow was collected from mouse femurs and tibias. Flt3L-expanded spleen cDCs were purified with anti-CD11c immunomagnetic beads, by passing the cells through 2 consecutive positive selection columns (Miltenyi Biotec). Purity was routinely greater than $93 \%$, maturation was comparable to cDCs from splenocytes of unmanipulated mice (Supplemental Figure 4), and cell viability was greater than $97 \%$ by trypan blue exclusion. Immediately following isolation and just prior to the initiation of liver I/R, $1 \times 10^{7} \mathrm{WT}, I l 10^{-/}$, or $\operatorname{Tl} 9^{-/-}$freshly isolated spleen $\mathrm{cDCs}$ were injected i.v. into recipient mice. We chose to inject $1 \times 10^{7} \mathrm{cDCs}$ i.v., as the number that end up in the liver approximates the number of $\mathrm{cDCs}$ within the liver of an unmanipulated mouse (i.e., $5 \times 10^{4}$; data not shown).

Flow cytometry. Flow cytometry and cell sorting was performed on a FACSAria (BD Biosciences). Fc receptors were blocked with $1 \mu \mathrm{g}$ antiFcyRIII/II antibody (2.4G2; Monoclonal Core Facility, Sloan-Kettering Institute) per $10^{6}$ cells. Cells were stained with fluorescence-conjugated antibodies to CD11c (clone HL3), CD11b (clone M1/70), Ly6C (clone AL-21), Ly6G (clone 1A8), CD40 (clone 3/23), CD80 (clone B7-1), CD86 (clone B7-2), and IL-10R (clone 1B1.3a) (all from BD Biosciences); MHCII (clone M5/114.15.2; eBioscience); and F4/80 (clone BM8; Invitrogen). Apoptosis and necrosis of liver cDCs was determined by staining cells with fluorescence-conjugated Annexin V and propidium iodide (PI; BD Biosciences) for 15 minutes. Depletion of cDCs from bulk NPC cultures

(NPCs-cDCs) was accomplished via cell sorting. Post-sort purities were routinely greater than $96 \%$. Intracellular cytokine staining was assessed following treatment of isolated CD45+ NPCs with Brefeldin A ( $1 \mu \mathrm{l}$ per $10^{6}$ cells; BD Biosciences) for 6 hours, using PE anti-mouse IL-6 (MP5-20F3; BD Biosciences), TNF (MP6-XT22; BD Biosciences), or IL-10 (JES5-16E3; $\mathrm{BD}$ Biosciences). Appropriate isotype controls were used when necessary. Data was analyzed using FlowJo software (Tree Star).

In vitro coculture assays. WT hepatocytes were rendered necrotic by incubation at $60^{\circ} \mathrm{C}$ for 60 minutes. Flow cytometry confirmed that more than $98 \%$ of hepatocytes (side scatter high) were necrotic $\left(\mathrm{PI}^{+}\right)$. Necrotic hepatocytes were plated at $5 \times 10^{6}$ cells/well in 96-well plates containing serum-free media (RPMI 1640, containing $10 \mathrm{mM}$ Hepes, $100 \mathrm{U} / \mathrm{ml}$ penicillin, $100 \mu \mathrm{g} / \mathrm{ml}$ streptomycin, $2 \mathrm{mM}$ L-glutamine; Media Preparation Core Facility, Sloan-Kettering Institute). Supernatant was harvested after a 12 -hour incubation period at $37^{\circ} \mathrm{C}$ and was used as conditioned media in subsequent coculture assays. WT CD $45^{+} \mathrm{NPCs}$, NPCs-cDCs, or purified cDCs from Flt3L-treated WT or Tlr9 $9^{--}$mice were cultured at $10^{6} \mathrm{cells} / \mathrm{ml}$ or $5 \times 10^{6} \mathrm{cells} / \mathrm{ml}$ in standard RPMI 1640 media (Media Preparation Core Facility, Sloan-Kettering Institute) or in the presence of conditioned media for 18 hours. In additional experiments, iCpG (10 $\mu \mathrm{g} / \mathrm{ml}$; Invivogen) was added to cultures, or conditioned media was pretreated for 2 hour with DNAse I (100 $\mu \mathrm{g} / \mathrm{ml}$; Sigma-Aldrich) at $25^{\circ} \mathrm{C}$.

Assays for oxidative burst and cytokines. Measurement of oxidative burst as gauged by the conversion of dihydrorhodamine (DHR) 123 to its oxidized form, rhodamine (R) 123, was determined by flow cytometry, using the Phagoburst Kit (Orpegen) according to the manufacturer's protocol. Ischemic liver $\mathrm{CD} 45^{+} \mathrm{NPCs}$ were cultured for 18 hours at a concentration of $10^{6} \mathrm{cells} / \mathrm{ml}$ in RPMI media containing $10 \%$ FCS. Supernatant and serum cytokine levels were determined using a cytometric bead array (Mouse Inflammation Kit; BD Biosciences). None of the tested cytokines were detected in control wells containing conditioned media only (data not shown).

Statistics. All $P$ values were generated from an ANOVA model representing the factorial design that was used in the corresponding experiment and were adjusted for multiple comparisons using simulation. This adjustment computes the adjusted $P$ values from the simulated distribution of the maximum absolute value of a multivariate $t$ random vector. $P$ values of less than 0.05 were considered significant.

\section{Acknowledgments}

We are grateful to the Laboratory of Comparative Pathology (Sloan-Kettering Institute) for their technical assistance with histopathology and measurement of serum ALT and to Mithat Gonen for his assistance with statistical analyses. This work was supported by NIH grants DK068346 and AI70658 (to R.P. DeMatteo) and the Marshall K. Bartlett Fellowship, Massachusetts General Hospital (to Z.M. Bamboat).

Received for publication May 28, 2009, and accepted in revised form December 2, 2009.

Address correspondence to: Ronald P. DeMatteo, Memorial SloanKettering Cancer Center, Box 203, 1275 York Avenue, New York, New York 10065, USA. Phone: (212) 639-3976; Fax: (212) 6394031; E-mail: dematter@mskcc.org.

\footnotetext{
1. Lotze MT, et al. The grateful dead: damage-associated molecular pattern molecules and reduction/oxidation regulate immunity. Immunol Rev. 2007;220:60-81.

2. Clavien PA, Harvey PR, Strasberg SM. Preservation and reperfusion injuries in liver allografts. An overview and synthesis of current studies. Transplanta-

tion. 1992:53(5):957-978.

3. Scaffidi P, Misteli T, Bianchi ME. Release of chromatin protein HMGB1 by necrotic cells triggers inflammation. Nature. 2002;418(6894):191-195.

4. Cavassani KA, et al. TLR3 is an endogenous sensor of tissue necrosis during acute inflammatory events. J Exp Med. 2008;205(11):2609-2621.
}

5. Millar DG, et al. Hsp70 promotes antigen-presenting cell function and converts T-cell tolerance to autoimmunity in vivo. Nat Med. 2003;9(12):1469-1476.

6. Colletti LM, et al. Role of tumor necrosis factoralpha in the pathophysiologic alterations after hepatic ischemia/reperfusion injury in the rat. J Clin Invest. 1990;85(6):1936-1943. 
7. Camargo CA Jr, Madden JF, Gao W, Selvan RS, Clavien PA. Interleukin- 6 protects liver against warm ischemia/reperfusion injury and promotes hepatocyte proliferation in the rodent. Hepatology. 1997;26(6):1513-1520.

8. Yamaguchi Y, et al. Monocyte chemoattractant protein-1 enhances expression of intercellular adhesion molecule-1 following ischemia-reperfusion of the liver in rats. Hepatology. 1998;27(3):727-734.

9. Jaeschke H. Molecular mechanisms of hepatic ischemia-reperfusion injury and preconditioning. Am J Physiol Gastrointest Liver Physiol. 2003; 284(1):G15-G26.

10. Kato A, Yoshidome H, Edwards MJ, Lentsch AB. Reduced hepatic ischemia/reperfusion injury by IL-4: potential anti-inflammatory role of STAT6. Inflamm Res. 2000;49(6):275-279.

11. Yoshidome H, Kato A, Edwards MJ, Lentsch AB. Interleukin-10 inhibits pulmonary NF-kappaB activation and lung injury induced by hepatic ischemia-reperfusion. Am J Physiol. 1999;277(5 Pt 1):L919-L923.

12. Yoshidome H, Kato A, Miyazaki M, Edwards MJ, Lentsch AB. IL-13 activates STAT6 and inhibits liver injury induced by ischemia/reperfusion. Am J Pathol. 1999;155(4):1059-1064.

13. Morelli AE, Thomson AW. Dendritic cells: regulators of alloimmunity and opportunities for tolerance induction. Immunol Rev. 2003;196:125-146.

14. Lu L, et al. Propagation of dendritic cell progenitors from normal mouse liver using granulocyte/ macrophage colony-stimulating factor and their maturational development in the presence of type- 1 collagen. J Exp Med. 1994;179(6):1823-1834.

15. Plitas G, et al. Dendritic cells are required for effective cross-presentation in the murine liver. Hepatology. 2008;47(4):1343-1351.

16. Loi $\mathrm{P}$, et al. The fate of dendritic cells in a mouse model of liver ischemia/reperfusion injury. Transplant Proc. 2004;36(5):1275-1279.

17. Lu L, et al. Growth of donor-derived dendritic cells from the bone marrow of murine liver allograft recipients in response to granulocyte/macrophage colony-stimulating factor. J Exp Med. 1995; 182(2):379-387.

18. Bamboat ZM, et al. Human liver dendritic cells promote T cell hyporesponsiveness. J Immunol. 2009; 182(4):1901-1911.

19. Plitas G, Burt BM, Nguyen HM, Bamboat ZM, DeMatteo RP. Toll-like receptor 9 inhibition reduces mortality in polymicrobial sepsis. J Exp Med. 2008;205(6):1277-1283.

20. Leadbetter EA, Rifkin IR, Hohlbaum AM, Beaudette BC, Shlomchik MJ, Marshak-Rothstein A. Chromatin-IgG complexes activate B cells by dual engagement of IgM and Toll-like receptors. Nature. 2002;416(6881):603-607.

21. Krieg AM, Vollmer J. Toll-like receptors 7, 8, and 9: linking innate immunity to autoimmunity. Immunol Rev. 2007;220:251-269.

22. Yasuda K, et al. Endosomal translocation of vertebrate DNA activates dendritic cells via TLR9-dependent and -independent pathways. J Immunol. 2005; 174(10):6129-6136.

23. Hornung V, et al. AIM2 recognizes cytosolic dsDNA and forms a caspase-1-activating inflammasome with ASC. Nature. 2009;458(7237):514-518.

24. Jaeschke H, Bautista AP, Spolarics Z, Spitzer JJ. Superoxide generation by neutrophils and Kupffer cells during in vivo reperfusion after hepatic ischemia in rats. J Leukoc Biol. 1992;52(4):377-382.
25. Auffray C, et al. CX3CR1+ CD115+ CD135+ common macrophage/DC precursors and the role of CX3CR1 in their response to inflammation. J Exp Med. 2009;206(3):595-606.

26. Daley JM, Thomay AA, Connolly MD, Reichner JS, AlbinaJE. Use of Ly6G-specific monoclonal antibody to deplete neutrophils in mice. J Lenkoc Biol. 2008; 83(1):64-70.

27. Geissmann F, Jung S, Littman DR. Blood monocytes consist of two principal subsets with distinct migratory properties. Immunity. 2003;19(1):71-82.

28. Boring L, Gosling J, Cleary M, Charo IF. Decreased lesion formation in CCR2 $2^{-/}$mice reveals a role for chemokines in the initiation of atherosclerosis. Nature. 1998;394(6696):894-897.

29. Izikson L, Klein RS, Charo IF, Weiner HL, Luster AD. Resistance to experimental autoimmune encephalomyelitis in mice lacking the $\mathrm{CC}$ chemokine receptor (CCR)2. J Exp Med. 2000;192(7):1075-1080.

30. Matzinger P. Tolerance, danger, and the extended family. Annu Rev Immunol. 1994;12:991-1045.

31. Jaeschke H, Lemasters JJ. Apoptosis versus oncotic necrosis in hepatic ischemia/reperfusion injury. Gastroenterology. 2003;125(4):1246-1257.

32. Morelli AE, et al. Internalization of circulating apoptotic cells by splenic marginal zone dendritic cells: dependence on complement receptors and effect on cytokine production. Blood. 2003; 101(2):611-620.

33. Stuart LM, Lucas M, Simpson C, Lamb J, Savill J, Lacy-Hulbert A. Inhibitory effects of apoptotic cell ingestion upon endotoxin-driven myeloid dendritic cell maturation. J Immunol. 2002;168(4):1627-1635.

34. Chen GY, Tang J, Zheng P, Liu Y. CD24 and Siglec-10 selectively repress tissue damage-induced immune responses. Science. 2009;323(5922):1722-1725.

35. Hemmi H, et al. A Toll-like receptor recognizes bacterial DNA. Nature. 2000;408(6813):740-745.

36. Bhan U, et al. TLR9 is required for protective innate immunity in Gram-negative bacterial pneumonia: role of dendritic cells. J Immunol. 2007; 179(6):3937-3946.

37. Barton GM. A calculated response: control of inflammation by the innate immune system. J Clin Invest. 2008;118(2):413-420.

38. Li M, et al. An essential role of the NF-kappa B/ Toll-like receptor pathway in induction of inflammatory and tissue-repair gene expression by necrotic cells. J Immunol. 2001;166(12):7128-7135.

39 . Georgel P, et al. Vesicular stomatitis virus glycoprotein $G$ activates a specific antiviral Toll-like receptor 4dependent pathway. Virology. 2007;362(2):304-313.

40. Yoshidome H, Kato A, Edwards MJ, Lentsch AB. Interleukin-10 suppresses hepatic ischemia/reperfusion injury in mice: implications of a central role for nuclear factor kappaB. Hepatology. 1999; 30(1):203-208.

41. Le Moine O, et al. Cold liver ischemia-reperfusion injury critically depends on liver T cells and is improved by donor pretreatment with interleukin 10 in mice. Hepatology. 2000;31(6):1266-1274.

42. Dinant S, Vetelainen RL, Florquin S, van Vliet AK, van Gulik TM. IL-10 attenuates hepatic I/R injury and promotes hepatocyte proliferation. J Surg Res. 2007;141(2):176-182.

43. Ivanov $S$, et al. A novel role for HMGB1 in TLR9mediated inflammatory responses to CPG-DNA. Blood. 2007;110(6):1970-1981.

44. Tian J, et al. Toll-like receptor 9-dependent activation by DNA-containing immune complexes is mediated by HMGB1 and RAGE. Nat Immunol. 2007; 8(5):487-496

45. Louis $\mathrm{H}$, et al. Hepatoprotective role of interleukin 10 in galactosamine/lipopolysaccharide mouse liver injury. Gastroenterology. 1997;112(3):935-942. 46. Louis $\mathrm{H}$, et al. Production and role of interleukin10 in concanavalin A-induced hepatitis in mice. Hepatology. 1997;25(6):1382-1389.

47. Tsung A, et al. Increasing numbers of hepatic dendritic cells promote HMGB1-mediated ischemiareperfusion injury. J Lenkoc Biol. 2007;81(1):119-128.

48. Pillarisetty VG, Miller G, Shah AB, DeMatteo RP. GM-CSF expands dendritic cells and their progenitors in mouse liver. Hepatology. 2003;37(3):641-652.

49. Miller G, Pillarisetty VG, Shah AB, Lahrs S, Xing Z, DeMatteo RP. Endogenous granulocyte-macrophage colony-stimulating factor overexpression in vivo results in the long-term recruitment of a distinct dendritic cell population with enhanced immunostimulatory function. J Immunol. 2002;169(6):2875-2885.

50. Heidenreich S, Gong JH, Schmidt A, Nain M, Gemsa D. Macrophage activation by granulocyte/ macrophage colony-stimulating factor. Priming for enhanced release of tumor necrosis factor-alpha and prostaglandin E2. J Immunol. 1989; 143(4):1198-1205.

51. Serbina NV, Pamer EG. Monocyte emigration from bone marrow during bacterial infection requires signals mediated by chemokine receptor CCR2. Nat Immunol. 2006;7(3):311-317.

52. Li L, et al. The chemokine receptors CCR2 and CX3CR1 mediate monocyte/macrophage trafficking in kidney ischemia-reperfusion injury. Kidney Int. 2008;74(12):1526-1537.

53. Dimitrijevic OB, Stamatovic SM, Keep RF, Andjelkovic AV. Absence of the chemokine receptor CCR2 protects against cerebral ischemia/reperfusion injury in mice. Stroke. 2007;38(4):1345-1353.

54. Martinon F, Mayor A, Tschopp J. The inflammasomes: guardians of the body. Annu Rev Immunol. 2009;27:229-265.

55. Imaeda AB, et al. Acetaminophen-induced hepatotoxicity in mice is dependent on Tlr9 and the Nalp3 inflammasome. J Clin Invest. 2009;119(2):305-314.

56. Meissner F, Molawi K, Zychlinsky A. Superoxide dismutase 1 regulates caspase-1 and endotoxic shock. Nat Immunol. 2008;9(8):866-872.

57. Kato A, Gabay C, Okaya T, Lentsch AB. Specific role of interleukin-1 in hepatic neutrophil recruitment after ischemia/reperfusion. Am J Pathol. 2002; 161(5):1797-1803.

58. Takeuchi $\mathrm{D}$, et al. Interleukin 18 causes hepatic ischemia/reperfusion injury by suppressing antiinflammatory cytokine expression in mice. Hepatology. 2004;39(3):699-710.

59. Dong X, Swaminathan S, Bachman LA, Croatt AJ, Nath KA, Griffin MD. Resident dendritic cells are the predominant TNF-secreting cell in early renal ischemia-reperfusion injury. Kidney Int. 2007; 71(7):619-628.

60. Rimoldi M, et al. Intestinal immune homeostasis is regulated by the crosstalk between epithelial cells and dendritic cells. Nat Immunol. 2005;6(5):507-514.

61. Tsung A, et al. The nuclear factor HMGB1 mediates hepatic injury after murine liver ischemia-reperfusion. J Exp Med. 2005;201(7):1135-1143.

62. Pillarisetty VG, Shah AB, Miller G, Bleier JI, DeMatteo RP. Liver dendritic cells are less immunogenic than spleen dendritic cells because of differences in subtype composition. JImmunol. 2004;172(2):1009-1017. 